\title{
Phase Locked High Power X-band Microwave Sources
}

\author{
Philip MacInnes, Craig R. Donaldson, Amy J. MacLachlan, \\ Colin G. Whyte, K. Ronald, Alan D. R. Phelps and \\ Adrian W. Cross \\ Department of Physics, SUPA, University of Strathclyde, \\ Glasgow G4 ONG, Scotland, UK
}

The numerical study of a low confining-field $(\sim 0.3 \mathrm{~T}) \mathrm{X}$-band Backward Wave Oscillator (BWO), suitable for implementation as the microwave source(s) in a phasesynchronous array, is presented. Analytical theory and CST Particle Studio simulations have been used to design a $\sim 9.6 \mathrm{GHz}$ BWO, generating $120 \mathrm{MW}, 1 \mathrm{~ns}$, pulses when driven by a $4 \mathrm{~ns}, 210 \mathrm{kV}, 1.5 \mathrm{kA}$ Radan 303B modulator.

Synchronisation of two (or more) such X-band BWOs may be achieved through independent adjustment of the fast risingedges of the applied potentials, allowing for tuning of the relative phase of the output pulses. With proper alignment this provides peak output intensities on the order of $\mathrm{N}^{2}$, from an array of $\mathrm{N}$ sources, improving range, signal-to-noise, etc. and facilitates "beam-stearing" of the combined output.

The experimental assembly will employ a single modulator, feeding two electron accelerating diodes, each incorporating a saturable Ferrite Spiral Line (FSL) [1]. Each FSL will be independently, externally, biased by a solenoidal magnetic field. COMSOL has been used to design the variable delay FSL, with the external bias adjusting the saturation time of the ferrite; this alters the rising-edge of the applied voltage pulse, changing the relative phase of the emitted radiation pulse. Active adjustment of the solenoid current allows for synchronization of the output across multiple BWOs.

Plans for construction of two X-band phase locked BWOs will be presented. The goals of the project are to carry out a systematic study of jitter [2] and to investigate phase locking of two X-band BWO sources based on cold cathodes.

\section{References}

[1] M.R. Ul'maskulov, S.A. Shunailov, M.I. Yalandin, K.A. Sharypov, A.G. Sadykova and V.G. Shpak "Ferrite Spiral Lines in Adjustable Synchronization of Nanosecond HighVoltage Generators", IEEE Transactions on Plasma Science, 47, N0. 10, October 2019.

[2] B.W. Hoff, D. M. French, D. S. Simon, P. D. Lepell, T. Montoya, and S. L. Heidger, "High current nonlinear transmission line based electron beam driver," Physical Review Accelerators and Beams, 20, 100401 (pp1 to 8), 2017.

\footnotetext{
Acknowledgements

* The support of the European Office of Aerospace Research and Development (EOARD) and the Air Force Office of Scientific Research (AFOSR) award FA8655-20-1-7011 is gratefully acknowledged
} 